\title{
On the field dependence of the vortex core size
}

\author{
V. G. Kogan and N. V. Zhelezina \\ Ames Laboratory - DOE and Department of Physics and Astronomy, Iowa State University, Ames IA 50011-3160
}

\begin{abstract}
We argue that in clean high- $\kappa$ type II superconductors, the low temperature vortex core size (defined as the coherence length $\xi$ ) in high fields should decrease with increasing applied field in qualitative agreement with experimental data. Calculations are done for the Fermi sphere and cylinder (with the field parallel to the cylinder axis). The results for clean materials at $T=0$ can be represented as $\xi(H) / \xi\left(H_{c 2}\right)=U\left(H / H_{c 2}\right)$ with $U$ being an universal function.

PACS numbers: $74.20 .-\mathrm{z}, 74.60 . \mathrm{w}, 74.60 . \mathrm{Ec}$
\end{abstract}

\section{INTRODUCTION}

The coherence length $\xi$ has first been introduced as a phenomenological length scale in the near- $T_{c}$ GinzburgLandau (GL) description where it sets, among other things, the "vortex core size" $\rho_{c}$. Since the core, in fact, does not have a sharp boundary, the size $\rho_{c}$ cannot be unambiguously defined and is commonly chosen "operationally convenient", i.e., in a way which varies from one experimental or theoretical situation to another. For example, $\rho_{c}$ may be defined as the radius of a circle where the persistent current is maximum, ${ }^{1}$ or - within the London approach - as the distance at which the divergent London current density reaches the depairing value, or assuming the core being a normal cylinder and equating the core contribution to the vortex energy to $\left(H_{c}^{2} / 8 \pi\right) \pi \rho_{c}^{2}$ with $H_{c}^{2} / 8 \pi$ being the condensation energy. ${ }^{2,3}$ Another example comes from the scanning tunneling work where "the vortex core radius is arbitrary defined by that distance $\rho_{c}$ from the vortex center, for which the tunneling current has decreased from $I_{\max }$ to $36 \%$ of $I_{\max }-I_{\min } . " 4$ Clearly, these procedures yield different values of $\rho_{c}$, although all of them have the same order of magnitude.

A lot of experimental effort has been invested recently in study of the vortex core size, see the review by Sonier and references therein. ${ }^{1}$ Notably, whatever the definition of $\rho_{c}$ is adopted, the low temperature $\rho_{c}$ is shown to decrease with increasing field in a number of materials such as $\mathrm{NbSe}_{2}, \mathrm{~V}_{3} \mathrm{Si}, \mathrm{LuNi}_{2} \mathrm{~B}_{2} \mathrm{C}, \mathrm{YBa}_{2} \mathrm{Cu}_{3} \mathrm{O}_{7-\delta}, \mathrm{CeRu}_{2}$ physical characteristics of which have little to do with each other (except all of them have a large GL parameter $\left.\kappa=\lambda_{L} / \xi\right)$. The dependences $\rho_{c}(H)$ for all tested materials are qualitatively similar; for large fields $\rho_{c} \sim 1 / \sqrt{H}$. Properties of the quasiparticle spectrum inside and outside the cores (where the excitations may form narrow conducting bands) are considered responsible for the $H$ and $T$ dependences of $\rho_{c}$.

The point of this paper is to provide a general theoretical argument based on the weak-coupling BCS theory in addressing causes of the field dependence of $\rho_{c}$. This argument is omitted in the current discussion of the problem in the experimental community, although a few examples of numerical solutions of the microscopic equations of superconductivity show that the $H$ dependence of $\rho_{c}$ follows from the theory under very general assumptions. ${ }^{5-7}$ Our approach can be applied to the problem of $\rho_{c}(H)$ only in large fields of high- $\kappa$ materials; still, we obtain our main results analytically that has certain advantages as compared to however powerful numerical techniques and enables one to make experimentally verifiable predictions.

We begin with the notion that within the microscopic theory, at arbitrary magnetic fields $H$ and temperatures $T$, it is not clear what exact value one should assign to $\xi$. The difficulty comes from the fact that $\xi$ and $\rho_{c}$ are not among the basic input parameters of the theory; instead, they should be calculated, analytically a very difficult if at all possible task in general. There is, however, a region at the second order phase transition from superconducting to normal state, the SN boundary, where the theory can be linearized and consequently $\xi$ is well defined.

The linearization has been performed in the seminal work of Helfand and Werthamer (HW) on the upper critical field $H_{c 2}(T, \tau)$ with $\tau$ being the mean scattering time on nonmagnetic impurities. ${ }^{8}$ They have shown that at $H_{c 2}$ where the order parameter $\Delta$ goes to zero, it satisfies for any $T$ a linear equation,

$$
-\xi^{2}(T) \Pi^{2} \Delta=\Delta,
$$

where $\boldsymbol{\Pi}=\nabla+2 \pi i \boldsymbol{A} / \phi_{0}$ is the gauge invariant gradient, $\boldsymbol{A}$ is the vector potential, and $\phi_{0}$ is the flux quantum. Formally, the equation is equivalent to the Schrödinger equation for a charge in uniform magnetic field; the field $H_{c 2}$ at which superconductivity first nucleates corresponds to the lowest eigenvalue of this equation: $H_{c 2}=\phi_{0} / 2 \pi \xi^{2}$. This field (and $\xi$ ) is obtained by solving the basic self-consistency equation of the theory:

$$
\frac{\hbar}{2 \pi T} \ln \frac{T_{c}}{T}=\sum_{\omega=0}^{\infty}\left(\frac{1}{\omega}-\frac{2 \tau S}{\beta-S}\right),
$$

where the function $S(T, \xi, \tau)$ can be written as:

$$
\begin{aligned}
S & =\frac{2 \beta}{\ell q} \int_{0}^{\infty} d s e^{-s^{2}} \tan ^{-1} \frac{s \ell q}{\beta} \\
& =\sum_{j=0}^{\infty} \frac{j !}{2 j+1}\left(-\frac{\ell^{2} q^{2}}{\beta^{2}}\right)^{j}, \\
\beta & =1+2 \omega \tau, \quad q^{2}=2 \pi H_{c 2} / \phi_{0}=\xi^{-2} .
\end{aligned}
$$


Here, $\omega=\pi T(2 n+1) / \hbar, n$ is an integer, $v$ is the Fermi velocity, and $\ell=v \tau$ is the mean-free path. The power series representation of $S$ is obtained by formally expanding $\tan ^{-1}$ and then integrating over $s$. The evaluation of $S$ was performed for the isotropic Fermi surface, i.e., for a Fermi sphere.

Thus, strictly speaking, the length $\xi$ is defined only at the SN phase boundary $H_{c 2}(T)$, and the question remains whether or not the same definition of $\xi$ is useful out of the immediate vicinity of $H_{c 2}(T)$. In fact, in a variety of situations (small samples, proximity systems) the SN transition may take place far from the bulk $H_{c 2}(T)$. To approach the problem of the phase boundary in these systems, one has to know $\xi(H, T)$ in a broad domain of the $H-T$ plane away of the bulk $H_{c 2}(T)$.

A method to evaluate $\xi(H, T)$ had been developed in Refs. 9 and 10. In principle, the method follows $\mathrm{HW}$ by utilizing the field uniformity and the $\Delta$ smallness at the SN transition wherever it occurs. Below, we outline the method as applied to the three-dimensional (3D) isotropic case of a Fermi sphere. Then, we consider 2D isotropic materials, i.e., the Fermi cylinder. We find that $\xi(H, T)$ so obtained decreases when $H$ increases toward $H_{c 2}$ away of the critical temperature $T_{c}$ in clean superconductors; the effect is suppressed by impurity scattering and is absent in the dirty limit. We provide a closed form equation for $\xi(H)$ for zero- $T$ clean case for both the Fermi sphere and Fermi cylinder, and show that the results can be represented as

$$
\frac{\xi(H)}{\xi\left(H_{c 2}\right)}=U\left(\frac{H}{H_{c 2}}\right)
$$

with $U$ being an universal function.

We next argue that the same procedure can be applied to the mixed state in applied fields $H<H_{c 2}$ near the vortex core centers in materials with large $\kappa=\lambda_{L} / \xi$. This is because near the centers the field (varying on the scale of the London penetration depth $\lambda_{L}$ ) can be taken as uniform and $\Delta(r) \rightarrow 0$ at the center. We find our results in qualitative agreement with the data available, uncertainties of experimental procedures of extracting $\xi$ notwithstanding.

\section{FERMI SPHERE: $H_{c 2}(T)$ AND $S(H, \xi, \omega, \ell)$}

Here we reproduce major points of the $\xi(H)$ derivation for the system near the second order SN transition with the help of the quasiclassical Eilenberger formalism. ${ }^{11}$ The main equations of the theory read:

$$
\begin{aligned}
& \tau \boldsymbol{v} \cdot \boldsymbol{\Pi} f=g(F+2 \tau \Delta)-(G+2 \omega \tau) f \\
& \frac{\Delta}{2 \pi T} \ln \frac{T_{c}}{T}=\sum_{\omega=0}^{\infty}\left(\frac{\Delta}{\hbar \omega}-F\right) .
\end{aligned}
$$

Here, $\boldsymbol{v}$ is the Fermi velocity; $f(\boldsymbol{r}, \omega, \boldsymbol{v})$ and $g(\boldsymbol{r}, \omega, \boldsymbol{v})$ are the Eilenberger Green's functions with averages over the Fermi surface denoted as $F=\langle f\rangle$ and $G=\langle g\rangle$.
In the normal phase $f=0$ and $g=1$. In a small vicinity of the SN transition, $|f| \ll 1$, whereas $g$ can still be set unity in linear approximation in $f$ due to normalization $g=\left(1-f f^{\dagger}\right)^{1 / 2}$ (for the same reason we do not need here an equation for $\left.f^{\dagger}\right)$. Equation (7) can be linearized:

$$
\begin{aligned}
& \ell \cdot \boldsymbol{\Pi} f=\tilde{F}-\beta f \\
& \ell=\boldsymbol{v} \tau, \quad \tilde{F}=F+2 \tau \Delta / \hbar, \quad \beta=1+2 \omega \tau .
\end{aligned}
$$

The solution of Eq. (9) is written as

$$
f=(\beta+\ell \cdot \boldsymbol{\Pi})^{-1} \tilde{F}=\int_{0}^{\infty} d \rho e^{-\rho(\beta+\ell \cdot \Pi)} \tilde{F},
$$

or for the Fermi surface average:

$$
F=\int_{0}^{\infty} d \rho e^{-\rho \beta}\left\langle e^{-\rho \ell \cdot \Pi} \tilde{F}\right\rangle .
$$

We now assume that $\Delta, F$, and $\tilde{F}$ satisfy Eq. (1); then, utilizing commutators of the operator $\boldsymbol{\Pi}$ in an uniform field and the known properties of exponential operators, ${ }^{9}$ one can manipulate Eq. (12) to

$$
F(\boldsymbol{r}, \omega)=\Delta(\boldsymbol{r}) \frac{2 \tau S}{\beta-S},
$$

where

$$
\begin{aligned}
S & =\sum_{m, j=0}^{\infty} \frac{\left(-q^{2}\right)^{j}}{j !(2 m+2 j+1)}\left(\frac{(m+j) !}{m !}\right)^{2}\left(\frac{\ell^{2}}{\beta^{2}}\right)^{m+j} \\
& \times \prod_{i=1}^{m}\left[(2 i-1) q^{2}-\xi^{-2}\right], \quad q^{2}=\frac{2 \pi H}{\phi_{0}}
\end{aligned}
$$

After substituting $F$ of Eq. (13) in the self-consistency Eq. (8) and cancelling $\Delta(\boldsymbol{r})$, we obtain an implicit Eq. (2) for $\xi(H, T, \ell)$. It is easy to see that at $H_{c 2}$ where $q^{2}=$ $1 / \xi^{2}$, the series (14) reduces to the HW sum (4).

The double sum (14) is in fact an asymptotic series and is difficult to deal with when the goal is to solve Eq. (2) for $\xi(H, T, \ell)$. The situation simplifies greatly in the dirty limit: $S$ is an expansion in powers of $\ell$. Keeping only the terms with $m+j=0,1$, one obtains

$$
S=1-\frac{\ell^{2}}{3 \xi^{2} \beta^{2}}, \quad \ell^{4} / \xi^{4} \ll 1, \quad \ell^{4} q^{4} \ll 1 .
$$

When substituted in the self-consistency Eq. (2), this yields de Gennes-Maki dirty limit result for $\xi(T, \ell)$ and $H_{c 2}(T){ }^{2}$ Since the field $q^{2}$ does not enter $S$, the coherence length in the dirty limit is field independent. In other words, in the dirty limit, the coherence length at a given $T$ determined at the upper critical field, is the same at this $T$ for any $H$.

Formally similar situation takes place near the critical temperature $T_{c}$ where the truncation (15) is justified by smallness of $q^{2}$ and $\xi^{-2}$ (not of $\ell$ ). We conclude that near $T_{c}$ the coherence length is field independent for any 
$\ell$. We then expect the strongest $H$ dependence of $\xi$ to exist at low temperatures in clean materials.

Given the complexity of series (14), it is desirable to have an integral representation for $S$ better suited for analytic and numerical work. This had been done in Ref. 10 for the 3D case of a spherical Fermi surface. We refer the reader for details of this nontrivial procedure and provide here the result:

$$
\begin{aligned}
& S(u, \sigma)=\sqrt{\pi} \operatorname{Re} \int_{0}^{\infty} d s \frac{\left(1-u s^{2}\right)^{\sigma-1}}{\left(1+u s^{2}\right)^{\sigma}} \operatorname{erfc} s, \\
& u=\frac{\ell^{2} q^{2}}{\beta^{2}}, \quad \sigma=\frac{1}{2}\left(1+\frac{1}{\xi^{2} q^{2}}\right) ;
\end{aligned}
$$

$\operatorname{erfc} s=(2 / \sqrt{\pi}) \int_{s}^{\infty} d z \exp \left(-z^{2}\right)(\sigma$ here differs by the sign from that used in Ref. 10). Note that $\sigma=1$ at $H_{c 2}$; integration by parts in (16) gives the HW integral (3). One can check by formally expanding the integrand in powers of $u s^{2}$ and integrating over $s$, that the integral (16) can indeed be written as the series (14), see Appendix B in Ref. 10.

\section{FERMI CYLINDER}

The calculations of the previous section cannot be done for an arbitrary Fermi surface. Still, for some simple shapes it is possible. The simplest of those is the Fermi cylinder with the field parallel to the cylinder axis. Employing the same procedure outlined for the Fermi sphere, we arrive at

$$
S=\sum_{m, n=0}^{\infty} \frac{(-1)^{n} 2^{m}(2 m+2 n) !}{n !(m !)^{2}}\left(\frac{u}{4}\right)^{m+n}(1-\sigma)_{m},
$$

where $u$ and $\sigma$ are defined in Eq. (17); we use the notation $(1-\sigma)_{m}=(1-\sigma)(2-\sigma) \cdots(m-\sigma) \cdot{ }^{12}$ The integral representation of this sum can be obtained in a manner similar to that described in Ref. 10 for the 3D case:

$$
S(u, \sigma)=\frac{2}{\sqrt{\pi}} \operatorname{Re} \int_{0}^{\infty} d s \frac{\left(1-u s^{2}\right)^{\sigma-1}}{\left(1+u s^{2}\right)^{\sigma}} e^{-s^{2}} ;
$$

see Appendix A. To verify this result we write:

$$
\begin{array}{r}
\frac{\left(1-s^{2} u\right)^{\sigma-1}}{\left(1+s^{2} u\right)^{\sigma}}=\left(\frac{1-s^{2} u}{1+s^{2} u}\right)^{\sigma-1} \frac{1}{1+s^{2} u} \\
=\sum_{m}\left(\begin{array}{c}
\sigma-1 \\
m
\end{array}\right)\left(-\frac{2 u s^{2}}{1+u s^{2}}\right)^{m} \frac{1}{1+s^{2} u} \\
=\sum_{m, n} \frac{(-1)^{\mu} \mu !(\sigma-1)_{m}}{(m !)^{2} n !} 2^{m} u^{\mu} s^{2 \mu},
\end{array}
$$

where $\mu=m+n$. Substituting this to Eq. (19) and integrating over $s$ one obtains $S$ in the form (18).

Having the quantity $S(H, \xi, T, \ell)$ for both 3D (Fermi sphere) and 2D (Fermi cylinder), one can solve Eq. (2) for $\xi(H, T, \ell)$ that in general can be done numerically. As pointed out above, the most interesting is the situation at $T=0$ in clean materials; this case can be treated analytically.

\section{CLEAN MATERIALS AT $T=0$}

To deal with the divergence of $\ln \left(T_{c} / T\right)$ in Eq. (2) we note than the sum over $\omega$ on the right-hand side (RHS) is actually extended to the Debye frequency $\omega_{D}$. Then, we have for the finite sum

$$
\sum_{\omega>0}^{\omega_{D}} \frac{1}{\hbar \omega} \approx \frac{1}{2 \pi T} \ln \frac{2 \hbar \omega_{D} e^{\gamma}}{\pi T}
$$

where the neglected terms are of the order $T^{2} / \hbar^{2} \omega_{D}^{2}$ and $\gamma \approx 0.577$ is the Euler constant. Hence the divergent $\ln T$ in Eq. (2) drops off. Since in the clean limit $\beta \approx 2 \omega \tau$ and $2 \tau S /(\beta-S) \approx S / \omega$ we obtain instead of Eq. (2) in the zero- $T$ limit:

$$
\ln \frac{2 \hbar \omega_{D}}{\Delta_{0}}=2 \pi T \sum_{\omega>0}^{\omega_{D}} \frac{S}{\hbar \omega} \rightarrow \int_{0}^{\omega_{D}} \frac{d \omega}{\omega} S(u, \sigma) .
$$

The integral at the RHS diverges logarithmically for $\omega_{D} \rightarrow \infty$, and so does the LHS; in other words, $\omega_{D}$ should drop off the result. This integral is evaluated in Appendix B for both 3D and 2D cases.

We then obtain an implicit equation for $\xi$ :

$\ln \frac{\hbar v q}{\Delta_{0} \alpha}+\frac{\cos (\pi \sigma)}{4}\left[\psi\left(\frac{1+\sigma}{2}\right)-\psi\left(\frac{\sigma}{2}\right)\right]+\frac{\psi(\sigma)}{2}=0$,

where $\psi$ is the Digamma function. The only difference between 2D and 3D situations is in the number $\alpha$ :

$$
\alpha_{2 D}=\sqrt{2}, \quad \alpha_{3 D}=e / \sqrt{2} .
$$

Setting $\sigma=1$ in Eq. (23) one obtains:

$$
H_{c 2}=\frac{\phi_{0}}{2 \pi \xi_{c 2}^{2}}, \quad \xi_{c 2}=\frac{\hbar v}{\Delta_{0} \sqrt{2} \alpha} e^{-\gamma / 2} .
$$

This yields for the 3D case:

$$
H_{c 2}(0)=\frac{\phi_{0} \Delta_{0}^{2}}{2 \pi \hbar^{2} v^{2}} e^{2+\gamma},
$$

the value obtained variationally by Gor'kov ${ }^{13}$ and proven to be exact by $\mathrm{HW}$; in $\mathrm{HW}$ reduced units it corresponds to $h^{*}(0)=H_{c 2}(0) / T_{c} H_{c 2}^{\prime}\left(T_{c}\right) \approx 0.72 .{ }^{8}$ For the $2 \mathrm{D}$ case, this gives $h^{*}(0) \approx 0.59$, the result obtained by Bulaevskii. ${ }^{14}$

We now observe that material parameters enter Eq. (23) only in the first term under the log-sign. If one measures the length in units of $\xi_{c 2}$ and uses the reduced field $h=H / H_{c 2}$, Eq. (23) takes the form independent of material parameters:

$$
\begin{aligned}
& \ln \left(2 h e^{\gamma}\right)+\frac{\cos (\pi \sigma)}{2}\left[\psi\left(\frac{1+\sigma}{2}\right)-\psi\left(\frac{\sigma}{2}\right)\right]+\psi(\sigma)=0 \\
& \sigma=\frac{1}{2}\left(\frac{1}{h \xi^{* 2}}+1\right), \quad \xi^{*}=\frac{\xi}{\xi_{c 2}} .
\end{aligned}
$$


Hence, this equation defines an universal curve $\xi^{*}(h)$ independent of either material characteristics $v_{F}, \Delta_{0}$ or the dimensionality. Given this curve and $H_{c 2}(0)$, one can recover $\xi(H)$ for a clean material at $T=0$.

The curve $\xi(H) / \xi\left(H_{c 2}\right)=U\left(H / H_{c 2}\right)$ is shown as a solid line in Fig. 1 for $0.15<H / H_{c 2}<1$; the reason why the small fields domain is not shown is given in the next section. Also shown are results of numerical evaluation of $\xi(H)$ for a few values of the impurity parameter $\lambda=$ $\hbar v / 2 \pi T_{c} \ell$. The numerical calculation is done with the help of the self-consistency Eq. (2) for arbitrary $T$ and $\lambda ; S\left(\xi, q^{2}, T, \lambda\right)$ is evaluated using an explicitly real form given in Appendix C.

It is worth noting that effect of raising temperature on $\xi(H)$ is qualitatively similar to that of the impurity scattering, see solid dots for $t=T / T_{c}=0.5$ : both suppress the field dependence of $\xi$. However, at low temperatures for reasonably clean materials in a broad domain of high fields, $\xi(H)$ is well represented by the zero- $T$ clean-limit curve; it is seen from the upper panel of Fig. 1 that for $\lambda=0.25$ this domain extends down to $h \approx 0.4$.

The lower panel of Fig. 1 shows the same results plotted against $1 / \sqrt{h}$, the quantity proportional to the intervortex spacing. In this manner the data are often presented to examine possible connection between the field dependence of the core size $\rho_{c}(H)$ and other properties of the mixed state. ${ }^{1}$ Our result shows that for materials on the clean side with $\lambda<1$, the slope $d \xi^{*} / d\left(h^{-1 / 2}\right)$ for $H \rightarrow H_{c 2}$ is universal. In fact, using Eq. (27) this slope at $H_{c 2}$ can be evaluated for the clean limit at $T=0$ :

$$
\left.\frac{d \xi^{*}}{d\left(h^{-1 / 2}\right)}\right|_{h=1}=1-\frac{8}{\pi^{2}} \approx 0.189 \text {. }
$$

We also observe that for real materials with $\lambda \neq 0$ and $T \neq 0, \xi^{*}(h)$ becomes flat as the field decreases with the impurity and temperature dependent plateaus.

\section{THE CORE SIZE}

The above discussion of $\xi(H)$ applies at the SN second order phase transition where the field is uniform and the order parameter $\Delta$ goes to zero. In fact, these conditions are met in vortex cores of high- $\kappa$ type-II superconductors in high fields. Indeed, in this case the field within the core of a size $\xi$ is practically uniform since it varies on a much larger scale $\lambda_{L}$. Besides, when one approaches the vortex center, $\Delta \rightarrow 0$. To evaluate $\xi$ in this situation, one can use the same formalism as at the SN phase boundary; in other words, the above procedure of evaluating $\xi(H)$ can be used to characterize the size $\rho_{c}$. The core size so defined is, of course, "operationally convenient" from our point of view just as various definitions mentioned in the Introduction. Our approach, however, is advantageous because along with evaluation of the $H$ dependence of the "core size" $\xi$, we predict that

- this dependence is weakened by scattering and disappears in the dirty limit,
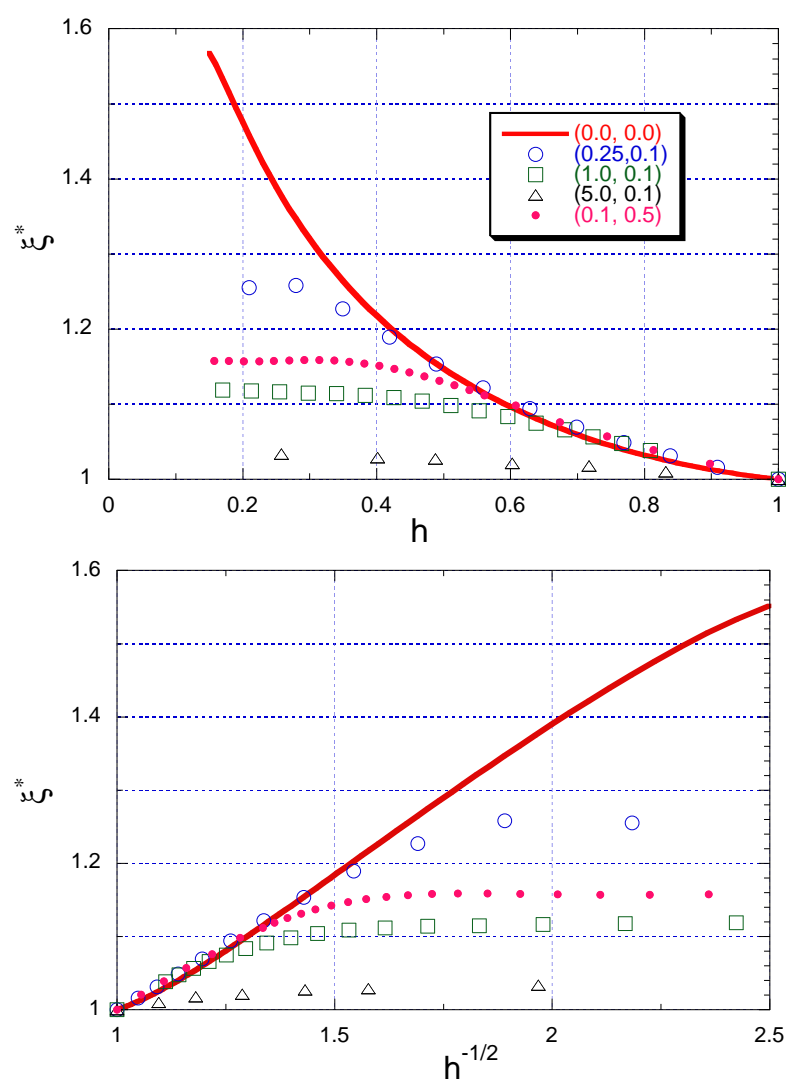

FIG. 1: (Color online) The upper panel: the normalized coherence length $\xi^{*}=\xi(H) / \xi\left(H_{c 2}\right)$ versus $h=H / H_{c 2}$. The solid line is calculated with the help of Eq. (27) for the clean limit at $T=0$. The open simbols show $\xi^{*}(\lambda, t, h)$ for a few values of the scattering parameter $\lambda=\hbar v / 2 \pi T_{c} \ell$ and reduced temperatures $t=0.1$ shown in pairs $(\lambda, t)$ on the legend. The full symbols are for a clean material at an elevated temperature: $(\lambda, t)=(0.1,0.5)$. The lower panel: $\xi^{*}$ versus $1 / \sqrt{h}$.

- the $H$ dependence of $\xi$ vanishes as $T \rightarrow T_{c}$,

- $\xi(H)$ is weakly affected by peculiarities of the Fermi surface, i.e., we expect qualitatively the same dependence for various materials,

- in reduced variables, the dimensionless coherence length $\xi^{*}=\xi / \xi_{c 2}$ should be nearly universal function of the reduced field $h=H / H_{c 2}$ for clean materials in high fields and low temperatures.

Therefore, our "theoretically convenient" definition of the core size can be checked experimentally.

One should put a note of caution on our claim of "universality" as stated in the last two points. This feature expressed in Eq. (27), has been derived for two simple Fermi surfaces, a sphere and a cylinder. ${ }^{15}$ Nevertheless, since the Fermi surface shape always enters calculations of macroscopic parameters as $\xi$ or $\lambda_{L}$ via averaging over the whole surface, one does not expect the fine features of the surface to alter drastically our conclusion (except 
in special circumstances, e.g., when the local density of states has sharp maxima at the surface). With this caveat we will use the term "universality" in further discussion.

There is another drawback to our approach. When applied to the SN phase boundary, say, of a proximity sandwich, the field $H$ in the $\xi(H)$ dependence is the externally controlled uniform applied field. Defining the core size as $\xi(H)$, we imply that $H$ is a the field value at the vortex center, $H_{0}$, which is nearly constant within the core provided $\kappa \gg 1$. However, the problem is that there is no reliable and generally applicable estimate of $H_{0}$, except numerical results with a particular choice of parameters for low- $\kappa$ and for high- $T_{c}$ materials. ${ }^{5-7}$ An exception is the case of isolated vortices (the applied field $\left.H_{a} \rightarrow 0\right)$ in the GL domain, where $H_{0} \approx 2 H_{c 1} \cdot{ }^{16}$ In a more interesting situation of $H_{a} \gg H_{c 1}$, the vortex fields are strongly overlapped, and variations of the actual field within the vortex lattice are weak relative to the applied field; in fact, they are on the order of $H_{c 1} \ll H_{a}$. Therefore, an error made by considering the field at the vortex axes as equal to the applied field is small. In other words, defining the vortex core size $\rho_{c}\left(H_{a}\right)$ as $\xi\left(H_{a}\right)$ has a reasonable chance of success only in large fields $H_{a} \gg H_{c 1}$ and improves as $H_{a} \rightarrow H_{c 2}$.

There is also a theoretical difficulty we encounter attempting to extend the analysis to $h \rightarrow 0$. In fact, the curve shown in Fig. 1 and generated by solving Eq. (27) shows oscillating behavior if extended to low fields beyond $h \approx 0.15 .{ }^{17}$ Our numerical work for finite $\lambda$ and $T$ shows that these oscillations are washed out quickly with increasing scattering and/or temperature and are hardly seen for $\lambda>0.25$, i.e., in still rather clean materials.

\section{COMPARISON WITH DATA}

Relating the results obtained to information available on the vortex core size, we focus on the $\mu \mathrm{SR}$ data reviewed recently by Sonier. ${ }^{1}$ This technique allows one to obtain the field distribution $h(\boldsymbol{r})$ within the vortex lattice. Then one can calculate the current distribution and define the core radius $\rho_{c}$ as the distance from the vortex axis to the current maximum in the nearest neighbor direction. This definition of the core size is independent of a model one may choose to theoretically describe the distribution $h(\boldsymbol{r})$, the point stressed in Ref. 18. We will consider here the data on so defined $\rho_{c}$.

The $\mu$ SR data on $h(\boldsymbol{r})$ can also be analyzed with the help of the London model or its nonlocal version. For simplicity, we consider here the standard London isotropic result:

$$
h(\boldsymbol{r})=B \sum_{\boldsymbol{G}} \frac{e^{i \boldsymbol{G} \cdot \boldsymbol{r}}}{1+\lambda_{L}^{2} G^{2}}
$$

where $B$ is the magnetic induction and the sum is extended over the reciprocal lattice $\boldsymbol{G}$.
It is relevant for this discussion that (a) the London model contains only one length scale, the penetration depth $\lambda_{L}$, and (b) the model implies the constant order parameter $\Delta$ and therefore breaks down at distances of the order $\xi$. The latter comes about formally in Eq. (29) since the sum is divergent (this is readily seen as the logarithmic divergence of $h$ when $r \rightarrow 0$ ). To mend this inherent shortcoming of the London model, various cutoffs are commonly used, e.g., by introducing a factor $\exp \left(-\right.$ const $\left.G^{2} \xi^{2}\right)$ which excludes distances smaller than $\xi$. Numerous efforts to fix the constant's value notwithstanding (see, e.g., Ref. 19), in practice this constant is used quite liberally depending on the application in question. Other cutoffs basically suffer of similar uncertainties. ${ }^{20}$ Hence, the reliable results of the London model are only those that are insensitive to the cutoff chosen. Still, one can fit the data $h(\boldsymbol{r})$ to a properly truncated sum (29), and extract the best-fit parameters $\lambda_{L}$ and $\xi$ along with their $H$ dependence. Interestingly enough, the so extracted $\xi(H)$ behaves as a function of $H$ in nearly the same manner as $\rho_{c}(H)$ extracted directly from the field distribution; it is found for a few materials that in high fields $\rho_{c} \approx \xi+C$ with a material dependent constant $C$. $^{1}$

We now consider the data on $\rho_{c}(H)$ for $\mathrm{V}_{3} \mathrm{Si}, \mathrm{NbSe}_{2}$, $\mathrm{YNi}_{2} \mathrm{~B}_{2} \mathrm{C}$, and $\mathrm{CeRu}_{2}$ provided in Refs. $18,21-23$, respectively, and summed up in the review by Sonier. ${ }^{1}$ All the samples are high quality single crystals and have large GL parameters $\kappa$; we assume them clean (the available scattering parameters are $\lambda\left(V_{3} S i\right) \approx 0.13$ and $\left.\lambda\left(\mathrm{NbSe}_{2}\right) \approx 0.15\right)$. The reduced temperatures of the $\mu \mathrm{SR}$ experiments were low: $\approx 0.22,0.33,0.19$, and 0.3 , respectively. For each material we have taken the $H_{c 2}$ at a corresponding temperature, calculated $\xi_{c 2}$, and normalized the experimental core radius to this value to obtain $\rho_{c}^{*}=\rho_{c} / \xi_{c 2}$. The results are plotted in the upper panel of Fig. 2 together with the theoretical $\xi^{*}$ versus reduced fields $h=H / H_{c 2}$. For reasons explained above we took only the data points for $h>0.15$. We expect the experimental $\rho_{c}^{*}(h)$ and the theoretical $\xi^{*}(h)$ to be shifted by a material, temperature and purity dependent constant: $\xi^{*}(h) \approx \rho_{c}^{*}(h)+C(\lambda, T)$. Since the temperatures and impurity parameters in different experiments were different, we do not expect these shifts to be the same for the materials examined. In this situation, we have chosen the constants $C$ so as to shift the data points as close as possible to our curve of $\xi^{*}(h)$. The result is shown in the middle panel of Fig. 2; the shifts needed are shown in the panel legend. Although the shifts vary, the data for different materials land nicely in a vicinity of our curve. This supports our guess of "universality", a considerable ambiguity of the procedure notwithstanding.

An interesting feature of the data and of the universal curve $\xi^{*}(1 / \sqrt{h})$ is seen in the lower panel of Fig. 2: the slope of this curve starting with the value $(28)$ of $\approx 0.2$ at $h=1$, increases to about 0.4 in the domain $0.25<$ $h<0.5$, and then drops back to about 0.2 near $h \approx 0.15$. In other words, the slope does not change much in each 

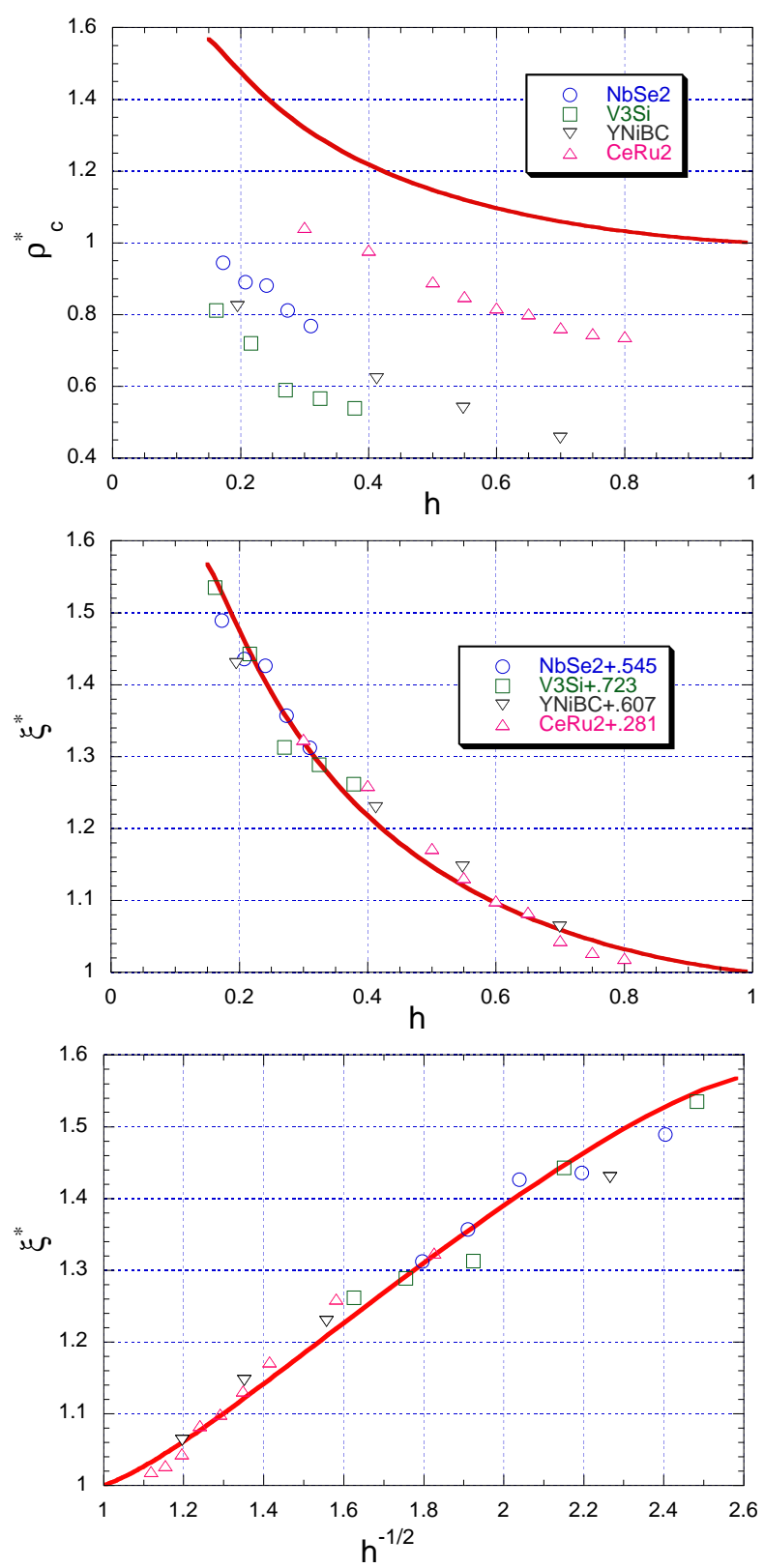

FIG. 2: (Color online) The upper panel: the experimental core radius $\rho_{c}$ normalized on $\xi_{c 2}=\sqrt{\phi_{0} / 2 \pi H_{c 2}(T)}$ of each compound for materials indicated in the legend; $T$ is the temperature of each experiment. The solid line is the theoretical $\xi^{*}(h)$ with $h=H / H_{c 2}(T)$, the same curve as in Fig. 1 . The middle panel: the same data shifted by amounts indicated in the legend. The lower panel: the same as the middle panel, but plotted versus $1 / \sqrt{h}$.

of these broad domains:

$$
\frac{d \xi^{*}}{d(1 / \sqrt{h})} \approx 0.2-0.4
$$

or in common units:

$$
\frac{d \xi}{d(1 / \sqrt{H})} \approx(0.2-0.4) \sqrt{\frac{\phi_{0}}{2 \pi}} .
$$

Since the measured core size $\rho_{c}$ differs from our $\xi$ by a constant shift, we may rewrite the last estimate as

$$
\frac{d \rho_{c}}{d(1 / \sqrt{H})} \approx(115-230) \AA \sqrt{k O e}
$$

in units employed in experiments. Given our suggestion of "universality", we expect the high-field slope $d \rho_{c} / d(1 / \sqrt{H})$ for all materials to be in this range. For the set of materials discussed here, this is the case: we roughly estimate the slope as 220 for $\mathrm{V}_{3} \mathrm{Si}, 190$ for $\mathrm{NbSe}_{2}$, 240 for $\mathrm{YNi}_{2} \mathrm{~B}_{2} \mathrm{C}$, and $170 \AA \sqrt{k O e}$ for $\mathrm{CeRu}_{2}$. The ability of our approach to provide the slope values in a good agreement with the data indicates that the model catches correctly the physics of the field dependence of the core size. The slopes are relevant in particular, given an uncertain relationship between $\xi$ we calculate and experimental $\rho_{c}$ (uncertain shifts $C$ in $\xi^{*}(h) \approx \rho_{c}^{*}(h)+C(\lambda, T)$ shown in the middle panel of Fig. 2).

Still, a number of questions remains to be addressed. Theoretically, it is not clear whether or not our clean limit results are compatible with the prediction of Kramer and Pesh that the core size of an isolated vortex defined as $\rho_{1}=\Delta /(\partial \Delta / \partial r)_{r \rightarrow 0}$ goes to zero as $T \rightarrow 0 .{ }^{24}$ We note, however, that our results for $\rho_{c}$ are meaningful only in large fields and for large GL parameters $\kappa$, whereas these authors have considered an isolated vortex in a material with $\kappa=0.9$. The same can be said with respect to calculations of Ichioka et al. done for the d-wave symmetry who find a shrinking core size in decreasing temperatures. ${ }^{25}$

Calculations of Miranović et al. of the low temperature field dependence of the length $\rho_{1}=\Delta_{m} /(\partial \Delta / \partial r)_{r \rightarrow 0}$ in the mixed state $\left(\Delta_{m}\right.$ is the order parameter maximum along the nearest neighbor direction) show that for clean materials with $\lambda<1$ the length $\rho_{1}(H)$ goes through a minimum and increases approaching $H_{c 2}$. It also shows a much stronger field dependence on the dirty side $(\lambda>$ 1) than our $\xi(H)$. A way out of this difficulty, in our opinion, is to conclude that $\rho_{1}(H)$ is not proportional either to our $\xi(H)$ or to existing data on the core size $\rho_{c}(H)$ in clean materials for which the minimum in $\rho_{c}(H)$ had not been recorded. ${ }^{1}$

We have to mention that our claim that the field dependence of $\xi$ and $\rho_{c}$ should be suppressed by impurity scattering and disappear altogether in the dirty limit contradicts calculations of Golubov and Hartmann done within the dirty limit Usadel formalism: they do find the $H$ dependence of $\rho_{c}$ for $\mathrm{NbSe}_{2}{ }^{4}$ Their conclusion have been questioned in Ref. 21 on the grounds that the dirty limit approximation does not hold for $\mathrm{NbSe}_{2}$ crystals. Moreover, Nohara et al. in Ref. 22 report that the $H$ dependence of $\rho_{c}$ extracted from the field dependence of the specific heat coefficient $\gamma$ and well pronounced in pure 
$\mathrm{NbSe}_{2}$, in fact disappears after doping the crystal with Ta. The doping changes the impurity parameter from 0.19 to 1.25 so that the observation is consistent with our conclusion. The data of this group on $\mathrm{YNi}_{2} \mathrm{~B}_{2} \mathrm{C}$ and $\mathrm{Y}\left(\mathrm{Ni}_{0.8} \mathrm{Pt}_{0.2}\right) \mathrm{B}_{2} \mathrm{C}$ are more convincing yet: the first crystal has the impurity parameter $\lambda \approx 0.4$ (i.e., it is on the clean side) whereas the Pt-doped crystal is on the dirty side with $\lambda \approx 2.4$; the $H$ dependence of $\rho_{c}$ in the doped crystal is practically absent. Still, the controversy remains and could be resolved if the $\mu \mathrm{SR}$ data were taken on a set of the same crystals with varying mean free path; candidates for such a study could be, e.g, the Co-doped $\mathrm{LuNi}_{2} \mathrm{~B}_{2} \mathrm{C}$ crystals. ${ }^{26}$

\section{Acknowledgments}

We are glad to thank J. Sonier for informative discussions and for providing a table of the published data. Thoughtful remarks of P. Miranović are greatly appreciated. Ames Laboratory is operated for the U. S. Department of Energy by Iowa State University under Contract No. W-7405-Eng-82. This work is supported by the Office of Basic Energy Sciences.

\section{APPENDIX A}

The sum (18) over $m, n$ can be replaced with the sum over $m$ from 0 to $\mu=m+n$ and the sum over $\mu$ from 0 to $\infty$; the former can be written as a hypergeometric function:

$$
S=\sum_{\mu=0}^{\infty}(2 \mu-1) ! !\left(-\frac{u}{2}\right)^{\mu}{ }_{2} F_{1}(-\mu, 1-\sigma ; 1 ; 2) .
$$

We now use the integral representation

$$
\begin{aligned}
{ }_{2} F_{1}(a, b ; c ; z)= & \frac{e^{-i \pi b} z^{1-c}}{4 \pi^{2}} \Gamma(c) \Gamma(1+b-c) \Gamma(1-b) \\
& \oint d t(t-z)^{c-b-1} t^{b-1}(1-t)^{-a},(\mathrm{~A} 2)
\end{aligned}
$$

where the contour circles the branch points at $t=0$ and $t=z$ twice in opposite directions; the representation holds everywhere except points where the $\Gamma$-factors diverge. ${ }^{27}$ This yields:

$$
\begin{array}{r}
S=\frac{e^{i \pi(\sigma-1)}}{4 \pi^{2}} \Gamma(1-\sigma) \Gamma(\sigma) \oint(t-2)^{\sigma-1} t^{-\sigma} \\
\sum_{\mu=0}^{\infty}(2 \mu-1) ! !\left[-\frac{u}{2}(1-t)\right]^{\mu} d t .
\end{array}
$$

The sum here is transformed to an integral with the help of identity

$$
\sum_{\mu=0}^{\infty}(2 \mu-1) ! !(-x)^{\mu}=\frac{2}{\sqrt{\pi}} \operatorname{Re} \int_{0}^{\infty} \frac{d s e^{-s^{2}}}{1+2 s^{2} x},
$$

which is proven by formally expanding $1 /\left(1+2 s^{2} x\right)$ in powers of $2 s^{2} x$ and integrating over $s$.

Then the contour integral emerges of the form

$$
J=\oint(t-2)^{\sigma-1} t^{-\sigma} \frac{d t}{1+s^{2} u(1-t)},
$$

which can be transformed back to the hypergeometric form after the substitution $t=v\left(1+s^{2} u\right) / s^{2} u=v z$ :

$$
J=\frac{4 \pi^{2} e^{i \pi(1-\sigma)}}{\Gamma(1-\sigma) \Gamma(\sigma)}{ }_{2} F_{1}(1,1-\sigma ; 1 ; 2 / z) .
$$

Further, ${ }_{2} F_{1}(1,1-\sigma ; 1 ; 2 / z)=(1-2 / z)^{\sigma-1}$ and we obtain Eq. (19) of the main text.

\section{APPENDIX B}

One has to evaluate the integral at the RHS of Eq.(22); we start with the Fermi cylinder:

$$
\int_{0}^{\omega_{D}} \frac{d \omega}{\omega} S=\frac{2}{\sqrt{\pi}} \operatorname{Re} \int_{0}^{\infty} d s e^{-s^{2}} \int_{0}^{\omega_{D}} \frac{d \omega}{\omega} \eta(\sigma, \omega),
$$

with

$$
\eta=\frac{\left(1-s^{2} u\right)^{\sigma-1}}{\left(1+s^{2} u\right)^{\sigma}}, \quad u=\frac{v^{2} q^{2}}{4 \omega^{2}}
$$

Substitution $x=\omega^{2} / \omega_{D}^{2}$ transforms the integral over $\omega$ to

$$
\begin{aligned}
& J=\frac{1}{2} \int_{0}^{1} d x \frac{(x-y)^{\sigma-1}}{(x+y)^{\sigma}} \\
& =\frac{(-1)^{-\sigma}(1+y)^{1-\sigma}}{4 y(\sigma-1)(2 y)^{-\sigma}}{ }_{2} F_{1}\left(1-\sigma, 1-\sigma ; 2-\sigma ; \frac{1+y}{2 y}\right) \\
& -\frac{(-1)^{\sigma}}{4}\left[\psi\left(\frac{1-\sigma}{2}\right)-\psi\left(1-\frac{\sigma}{2}\right)\right],
\end{aligned}
$$

where

$$
y=\frac{s^{2} v^{2} q^{2}}{4 \omega_{D}^{2}} \ll 1
$$

because large values of $s$ are cut off by $e^{-s^{2}}$. Utilizing the reflection formulas for the Digamma function, ${ }^{12}$ $\psi(1-z)=\psi(z)+\pi \cot (\pi z)$, the expression in square parentheses is rewritten as

$$
\psi\left(\frac{1+\sigma}{2}\right)-\psi\left(\frac{\sigma}{2}\right)-\frac{2 \pi}{\sin (\pi \sigma)} .
$$

We further use the asymptotic formula 15.3 .13 of Ref. 12 for ${ }_{2} F_{1}$ since $1 / 2 y \gg 1$. Then, the real part of $J$ assumes the form:

$$
\begin{array}{r}
\operatorname{Re} J=-\frac{\cos (\pi \sigma)}{4}\left[\psi\left(\frac{1+\sigma}{2}\right)-\psi\left(\frac{\sigma}{2}\right)\right] \\
-\frac{1}{2}[\ln 2+\gamma+\psi(\sigma)]+\ln \frac{2 \omega_{D}}{v q}-\ln s
\end{array}
$$


The integration over $s$ in Eq. (B1) is now straightforward: the $s$ independent part of $\operatorname{Re} J$ enters the result being unchanged because $(2 / \sqrt{\pi}) \int_{0}^{\infty} d s e^{-s^{2}}=1$. Further: $(2 / \sqrt{\pi}) \int_{0}^{\infty} d s e^{-s^{2}} \ln s=-\gamma / 2-\ln 2$. Collecting all terms in the self-consistency equation (22) we obtain Eq. (23). As expected, the large parameter $\omega_{D}$ cancels out from the final result.

For the 3D situation, we have to replace in 2D Eq. (B1) $(2 / \sqrt{\pi}) \int_{0}^{\infty} d s e^{-s^{2}} \operatorname{Re} J$ with $\sqrt{\pi} \int_{0}^{\infty} d s \operatorname{erfc}(s) \operatorname{Re} J$. As in $2 \mathrm{D}$, the $s$ independent part of Re $J$ enters the result being unchanged since $\sqrt{\pi} \int_{0}^{\infty} d s \operatorname{erfc}(s)=1$, whereas $\sqrt{\pi} \int_{0}^{\infty} d s \operatorname{erfc}(s) \ln s=-1-\gamma / 2$. This gives the $3 \mathrm{D}$ version of Eq. (23).

\section{APPENDIX C}

An explicitly real representation of the integral (16) is given in Ref. 10 for the Fermi sphere. Here we provide it for the 2D isotropic case of Eq. (19). To this end, one separates the integration domain in two: $0<s<1 / \sqrt{u}$ and $1 / \sqrt{u}<s<\infty$. In the first, the integration variable is changed to $y=s / \sqrt{u}$ whereas in the second to $y=$ $(s \sqrt{u})^{-1}$. Then we obtain:

$$
\begin{aligned}
S(u, \sigma) & =\frac{2}{\sqrt{\pi u}} \int_{0}^{1} d y \frac{\left(1-y^{2}\right)^{\sigma-1}}{\left(1+y^{2}\right)^{\sigma}} \\
& \times\left[\exp \left(-\frac{y^{2}}{u}\right)-\cos (\pi \sigma) \exp \left(-\frac{1}{y^{2} u}\right)\right],
\end{aligned}
$$

the form easy to deal with numerically.
1 J.E. Sonier, cond-mat/0404115.

2 P. DeGennes, Superconductivity of Metals and Alloys, New York, Addison-Wesley, 1989.

3 M.Tinkham, Introduction to Superconductivity, McGrawHill, 1996.

4 A.A. Golubov and U. Hartman, Phys. Rev. Lett. , 72, 3602 (1994).

5 M. Ichioka, A. Hasegawa, and K. Machida, Phys. Rev. B 59, 184 (1999).

6 M. Ichioka, A. Hasegawa, and K. Machida, Phys. Rev. B 59, 8902 (1999).

7 P. Miranović, M. Ichioka, and K. Machida, condmat/0312420.

8 E.H. Helfand, N.R. Werthamer, Phys. Rev. 147, 288 (1966).

${ }^{9}$ V.G. Kogan, Phys.Rev.B 32, 139 (1985).

10 V.G. Kogan and N. Nakagawa, Phys. Rev. B , 35, 1700 (1987). In this paper, $S$ has been used to calculate the SN phase boundary of a thin film in parallel field. The boundary conditions employed were later questioned by $\mathrm{P}$. Scotto and W. Pesh, J Low Temp. Phys. 84, 301 (1991) and by J. Hara and K. Nagai, J. Phys. Soc. Jpn. 63, 2331 (1994). The integral representation of $S$ of this paper is not related to boundary conditions and is correct.

11 G. Eilenberger, Z. Phys. 214, 195 (1968).

12 Handbook of Mathematical Functions, edited by M. Abramowitz and A. Stegun, Natl. Bur. Stand. Appl. Math. Ser. No. 55 (U.S. GPO, Washington, D.C., 1965).

13 L.P. Gor'kov, Zh. Exp. Teor. Fiz. 37, 833 (1959); Sov. Phys. JETP, 10, 593 (1960).

${ }^{14}$ L.N. Bulaevskii, Zh. Eksp. Teor. Fiz. 68, 1278 (1973); Sov. Phys JETP, 38, 634 (1974).

15 The same result can be shown to hold for a Fermi surface consisting of two sheets, a sphere and a cylinder with two different but constant gaps; the derivation will be published elsewhere. This model Fermi surface is proven to work well for $\mathrm{MgB}_{2}$; see, e.g., V.G. Kogan, Phys. Rev. B 66, 020509 (2002) and P. Miranović, K. Machida and V.G. Kogan, J. Phys. Soc. Jpn, 72, No.2, 221 (2003). The model is probably good for $\mathrm{NbSe}_{2}$ as well.

16 A.A. Abrikosov, Fundamentals of the Theory of Metals, North-Holland, New York, 1988.

17 The function $\xi^{*}(h)$ for $h<0.15$ oscillates with decreasing amplitude when $h \rightarrow 0$ where $\xi^{*} \rightarrow e^{\gamma / 2} \approx 1.33$. Formally, the oscillations are due to the branch point of the integrands (16) and (19) which produces $\cos (\pi \sigma)$ in the real part of the integral. The physical significance of this zero- $T$ clean limit behavior is unclear.

18 J.E. Sonier, F.D. Callaghan, R.I. Miller, E. Boaknin, L. Taillefer, R.F. Kiefl, J.H. Brewer, K.F. Poon, and J.D. Breweer, Phys. Rev. Lett. 93, 017002 (2004); condmat/0401271.

19 A. Yaouanc, P. Dalmas de Réotier, and E.H. Brandt, Phys. Rev. B 55, 11107 (1997).

20 J.R. Clem, J. Low Temp. Phys. 18, 427 (1975).

21 J.E. Sonier, R.F. Kiefl, J.H. Brewer, J. Chakhalian, S.R. Dunsiger, W.A. MacFarlane, R.I. Miller, A. Wong, G.M. Luke, and J.W. Brill, Phys. Rev. Lett. 79, 1742 (1997).

22 M. Nohara, M. Isshiki, F. Sakai, and H. Takagi, J. Phys. Soc Jpn. 68, 1078 (1999).

${ }^{23}$ R. Kadono, W. Higemoto, A. Koda, K. Ohishi, T. Yokoo, J. Akimitsu, M. Hedo, Y. Inada, Y. Ōnuki, E. Yamamoto, and Y. Haga, Phys. Rev. B 63, 224520 (2001).

${ }^{24}$ W. Pesh and L. Kramer, J. Low Temp. Phys. 15, 367 (1974).

25 M. Ichioka, N. Hayashi, N. Enomoto, and K. Machida, Phys. Rev. B 53, 15316 (1996).

${ }^{26}$ K.O. Cheon, I.R. Fisher, V.G. Kogan, P. Miranović, P.L. Gammel, and P.C. Canfield, Phys. Rev. B, 58, 6463 (1998).

27 P.M. Morse and H. Feshbach, Methods of Theoretical Physics, McGraw-Hill, 1953; v.1, Eq. (5.3.20). 